W. D. Strain · N. Chaturvedi $\cdot$ P. Nihoyannopoulos •

\author{
C. J. Bulpitt · C. Rajkumar · A. C. Shore
}

\title{
Differences in the association between type 2 diabetes and impaired microvascular function among Europeans and African Caribbeans
}

Received: 27 April 2005 / Accepted: 16 July 2005 / Published online: 29 September 2005

(C) Springer-Verlag 2005

\begin{abstract}
Aims/hypothesis: Diabetes is associated with microvascular damage in all populations, but diabetic patients of Black African descent (African Caribbeans) have a greater risk of vascular target organ damage than would be anticipated for any given blood pressure level. We investigated whether this may be due to differences in the microvasculature. Materials and methods: To assess the maximum hyperaemic response to heating and the post-ischaemic response, Laser Doppler fluximetry was performed on 51 and 100 Europeans, and on 66 and 88 African Caribbeans with and without diabetes, respectively. Subjects were aged between 40 and 65 years and recruited from the general population. Echocardiographic interventricular septal thickness (IVST) was measured as a proxy for vascular target organ damage. Results: In diabetic subjects of both ethnic groups, the maximum hyperaemic response and peak response to ischaemia were attenuated as compared to the corresponding non-diabetic subjects $(p=0.08$ for diabetic and 0.03 for non-diabetic Europeans; $p=0.03$ and 0.1 for African Caribbeans). Adjustment for cardiovascular risk factors, in particular
\end{abstract}

W. D. Strain $(\bowtie) \cdot$ N. Chaturvedi

International Centre for Circulatory Health,

Faculty of Medicine, Imperial College London at St Mary's, Norfolk Place,

London, W2 1PG, UK

e-mail: d.strain@imperial.ac.uk

Tel.: +44-207-5943646

Fax: +44-207-5943392

P. Nihoyannopoulos

Cardiology Department, Faculty of Medicine,

Imperial College London at the Hammersmith Hospital,

London, UK

C. J. Bulpitt · C. Rajkumar

Care of the Elderly, Faculty of Medicine,

Imperial College London at the Hammersmith Hospital, London, UK

\section{A. C. Shore}

Institute of Biomedical and Clinical Science,

Peninsula Medical School (Exeter),

Exeter, UK insulin and blood pressure, abolished these differences in Europeans ( $p=0.8$ for diabetic and 0.2 for non-diabetic Europeans), but not in African Caribbeans ( $p=0.03$ and 0.05). Conclusions/interpretation: Persisting microvascular dysfunction in African Caribbeans may contribute to the increased risk of target organ damage observed in diabetes in this population. The weak contribution of conventional cardiovascular risk factors to these disturbances indicates that conventional therapeutic interventions may be less beneficial in these patients. There was a risk-factor-independent, inverse association between IVST and maximal hyperaemia. These ethnic differences in microvascular responses to temperature and arterial occlusion could account for increased target organ damage in African Caribbeans.

Keywords African Caribbeans · Black Africans · Ethnicity · Europeans - Interventricular septal thickness · Laser Doppler fluximetry · Microvascular function . Type 2 Diabetes

Abbreviations AU: Arbitrary units - CARDS: Collaborative Atorvastatin Diabetes Study - IVST: Interventricular septal thickness · UKPDS: United Kingdom Prospective Diabetes Study

\section{Introduction}

Diabetes leads to premature cardiovascular morbidity and mortality, an effect largely independent of conventional cardiovascular risk factors [1]. People of Black African descent (African Caribbeans) are particularly susceptible to diabetes, with a prevalence three times that of populations of European origin [2]. The risk of vascular complications such as nephropathy, left ventricular hypertrophy and stroke is also higher than can be accounted for by rates of diabetes or other concomitant cardiovascular risk factors, such as hypertension [3-6].

The role of impaired microvascular haemodynamics is increasingly being recognised in the pathogenesis of sub- 
sequent vascular target organ damage [7, 8]. This may, in part, represent shared risk factors; however, the association remains when these are adjusted for, indicating that microvascular injury may occur on the mechanistic pathway between risk factors and vascular target organ damage. Diabetes is known to adversely affect the microvasculature, and this may be one of the factors leading to the increased risk of cardiovascular morbidity and mortality. In the United Kingdom Prospective Diabetes study (UKPDS), aggressive blood pressure control and tight glycaemic control were associated with an improvement in clinical microvascular outcomes $[9,10]$, suggesting that, at least to some degree, adverse effects of diabetes are mediated through these measures. However, the UKPDS population was predominantly white European, and it is uncertain whether these results can be extrapolated to a multi-ethnic population.

The role of ethnic differences in large vessel vascular function has been studied in the general population [11] and in people with type 2 diabetes [12], however the effect of diabetes on the microvessels $(<80 \mu \mathrm{m}$ in diameter) has not been explored. We cannot assume that abnormalities observed in the large vessels reflect those in the microcirculation, given the differences in structure, function and regulation of these vessels. We have previously demonstrated that patients of African Caribbean origin, drawn from the general population, have impaired microvascular functional responses to heating and ischaemic stimuli compared to an age- and sex-matched European sample [13]. These differences were independent of differences in baseline blood pressure and other conventional cardiovascular risk factors.

We hypothesised that microvascular structure and function would be compromised in diabetic patients of African Caribbean and white European descent, compared to control subjects without diabetes, and that explanatory factors for diabetes-related microvascular disturbances would differ by ethnicity. Further, we explored associations between microvascular measures and an index of vascular target organ damage, i.e. interventricular septal thickness (IVST), a proxy for left ventricular hypertrophy.

\section{Subjects and methods}

\section{Study population}

Explanations for the level of risk of a population need to be sought in representative population samples. Clinic populations are prone to referral bias, and exclusion of individuals due to prior disease, such as heart disease, would also result in a biased non-representative comparison, as these conditions are distributed differently by ethnicity. We therefore performed a cross-sectional study on a representative sample of the general population with and without diabetes selected from family practitioner registers in London, UK. This provides a comprehensive sampling frame, as more than $97 \%$ of the population is registered [14], and notification of diabetes status is a requirement under the family practi- tioner contract, and the English National Service Framework (http://www.dh.gov.uk/PolicyAndGuidance/Health AndSocialCareTopics/Diabetes/fs/en, accessed 4/07/05). People of Black African descent have migrated to the UK directly from West Africa, or via the Caribbean [15]. Our previous study demonstrated that all respondents were firstgeneration migrants, $91 \%$ were born in the Caribbean and $9 \%$ arrived directly from West Africa. In that study, blood pressures were identical in these two groups, and rates of end organ damage were similarly high $[16,17]$.

Men and women aged 40 to 65 years of African Caribbean and white European descent were stratified by age group, sex and ethnicity and then randomly allocated a unique study number. Random sampling by these strata was performed yielding 188 non-diabetic participants and 117 participants with type 2 diabetes. The response rate was $54 \%$ which did not vary by ethnicity or diabetes status. No matching on other cardiovascular risk factors was performed, as we wished to determine the degree to which these factors could account for any ethnic differences observed.

Only subjects with severe disease, such as cancer or a major psychiatric illness, or who were HIV- or hepatitis-Bpositive, were excluded from the sampling frame. Those who agreed to participate were telephoned for a final eligibility check, including a cross-check of age and ethnic group, and to determine the presence of sickle cell disease or atrial fibrillation, which interfere with vascular functional assessments and were therefore reasons for exclusion. Respondents were then invited to attend the Hammersmith Hospital for a clinical examination. The study protocol was approved by the medical research ethics committee of the Hammersmith Hospital.

All participants gave informed written consent and completed a questionnaire on demographic data, medical history, medications, and lifestyle behaviour. Ethnicity was self-assigned, and cross-checked against own and parental country of birth. Measures taken according to a standard protocol included height, weight and waist-to-hip ratio [18]. Blood samples were analysed by the on-site laboratory, which is a member of the appropriate UK National Quality Assessment Scheme. Glucose and lipid levels were determined using the AU600 Olympus Diagnostic analyser (Olympus Diagnostic Systems, Eastleigh, UK ) and insulin was determined by Abbott Axsym immunometric assay (Abbott Laboratories, Abbott Park, IL, USA). All patients reported as "without diabetes" received an oral glucose tolerance test to confirm this status. All participants attended in the morning, fasted from at least midnight the night before, having refrained from smoking, drinking tea or coffee and taking any medications.

\section{Cardiovascular assessment}

Supine resting blood pressure was measured three times after 5 min rest using an automated device (Omron 705CP; Omron Healthcare Europe, Hoofddorp, the Netherlands.). Echocardiography (2D) was performed to a standardised 
protocol [19]. Ambulatory blood pressure was measured over the subsequent $24 \mathrm{~h}$ (Duolter; Novocor, RueilMalmaison, France). All observers were trained in study measures, and repeatability was assessed periodically throughout the study.

While standard formulae to estimate left ventricular mass index are valid when comparing within an ethnic group, they may be less appropriate when comparing between ethnic groups, as the structural components of the ventricle respond differently to insults such as raised blood pressure [20-22]. As IVST has been shown to correlate well with changes in blood pressure across ethnic group comparisons [21], we report the directly measured IVST as a proxy for left ventricular hypertrophy and thus target organ damage.

\section{Microvascular assessment—rationale}

Although microvascular outcomes are easily identified within the retinal and renal vascular beds, it is difficult to study microvascular function within these beds in large numbers of people, and any invasive investigation may alter the function therein. Skin vessels are an easily accessible location to explore the microvasculature, can be investigated non-invasively and mirror disturbances in other vascular beds. These measures have previously been correlated with cardiovascular risk [23].

As the skin is a thermoregulatory organ, resting measurements exhibit a high degree of within-person variability, and dynamic responses to stimuli are preferred. We measured responses to heating and to ischaemia in arbitrary units (AU) with laser Doppler fluximetry (DRT4; Moor Instruments, Axminster, UK) using an established protocol [24].

\section{Response to heating}

A heated brass disc (area: $0.7 \mathrm{~cm}^{2}$ ) was fixed on a flat region, not overlying any large vessels, on the dorsum of the left foot. This was maintained at $42^{\circ} \mathrm{C}$ for $30 \mathrm{~min}$. The maximum hyperaemic response was assessed by measuring skin vessel flux (flow) at eight equidistant points in the disk area and averaging the results. Minimum microvascular resistance was calculated. The intra-subject, intraobserver variability over a period of 3 months or less was $8.2 \pm 2.0 \%$ within this study. This was measured in 12 individuals, three of whom had diabetes. The variability within a single subject over a 10 -year period is $10 \%$.

\section{Response to ischaemia}

A sphygmomanometer cuff was inflated to $30 \mathrm{mmHg}$ above ankle systolic blood pressure for $3 \mathrm{~min}$. During occlusion, the flux generated by Brownian motion of the interstitial fluid and intracellular fluid at the heated site was recorded (biological zero). At $3 \mathrm{~min}$ the occlusion was released prompting a reactive increase in flow. In an unheated area of skin the peak value of this flow was recorded. The intra-subject reproducibility of the peak response was $16.2 \pm 2.8 \%$.

\section{Sample size}

A standardised difference of 0.55 for a given variable with $80 \%$ power and $5 \%$ significance could be detected with around 50 individuals in each diabetes subgroup stratified by ethnicity.

\section{Statistical analysis}

The mean of the three resting blood pressures was used, and skewed variables were log transformed, with the exception of $\mathrm{HbA}_{1} \mathrm{c}$, which was not normalised by $\log$ transformation, but was inverse-transformed. Maximal hyperaemic response was corrected for the biological zero. Minimum microvascular resistance was calculated as the mean arterial blood pressure measured at the brachial artery divided by maximum flow. In exploring mechanisms for the effect of diabetes, we took account of potential confounding effects of age and statin use, as well as mechanistic factors. Mechanistic factors included blood pressure indices (brachial systolic, ankle systolic, brachial diastolic, mean arterial pressure, $24 \mathrm{~h}$ mean ambulatory blood pressure, day-time and night-time ambulatory systolic blood pressure), body size (height, weight, BMI, waist circumference and waist-to-hip ratio), insulin resistance (fasting glucose and fasting insulin) and lipid profile (cholesterol, cholesterol : HDL ratio, HDL and triglycerides). A single variable from each of these groups was included in the final model, based on the greatest increase in the amount of variance explained by their inclusion in the bivariate model. Separate models for each ethnic group were constructed, so that ethnicity-specific determinants of diabetic microvascular dysfunction could be reported. Statistical significance for categorical variables was calculated using the Chi square test and the Student's $t$-test for continuous variables. Analysis of co-variance was employed to determine explanations for ethnic differences in key variables, with tests for interaction. All variables were tested for sex/ethnicity and sex/diabetes interactions before data were pooled. A result was deemed significant if $p \leq 0.05$. In keeping with the recommendations of Cupples [25] and Rothman [26], the measured significance of the variables of interest is reported without adjustment for multiple testing. Bonferroni's adjustment is not used on the variables under investigation, as it is only relevant for additional variables within models. Analyses were performed using STATA (version 7) (StataCorp, College Station, TX, USA). Analysable microvascular recordings were available on 273 of 305 participants. The rest were discarded due to poor quality, either because of patient factors or technical difficulties or an incomplete dataset of other variables. There were no differences in the baseline 
characteristics between those included and those with incomplete datasets. The impact of adding additional variables in accounting for the variance of the model $\left(R^{2}\right)$ was tested by the likelihood ratio statistic. The variable that made the greatest impact was included in the final model.

\section{Results}

\section{Baseline characteristics}

People with diabetes were older than those without in both ethnic groups (Table 1), and, as anticipated, had a higher resting blood pressure, were more obese, had higher fasting insulin and fasting glucose. There was no difference in albumin excretion rate between any group. Total cholesterol and the total cholesterol : HDL-cholesterol ratio were more favourable in subjects with diabetes, probably be- cause of their higher use of 3-hydroxy-3-methylglutaryl coenzyme A reductase inhibitor (statin). Triglycerides, however, were higher and HDL-cholesterol was lower in diabetic than in non-diabetic subjects, but only in the European group. African Caribbeans had a more favourable lipid profile than Europeans, both with and without diabetes, despite lower statin usage. Duration, control and the pharmacological therapy of diabetes were similar in both ethnic groups. Smoking (current or ex) was more common in Europeans, but other lifestyle factors such as self-reported physical activity were similar between groups (data not shown). All groups were generally healthy, as would be expected from a general population sample, with only three participants without diabetes (all European) and seven with diabetes (four Europeans and three African Caribbeans) having a history of previous cardiovascular disease. IVST was greater in African Caribbeans, although this did not differ by diabetes status. There was no dif-

Table 1 Baseline characteristics stratified by ethnicity and diabetes status

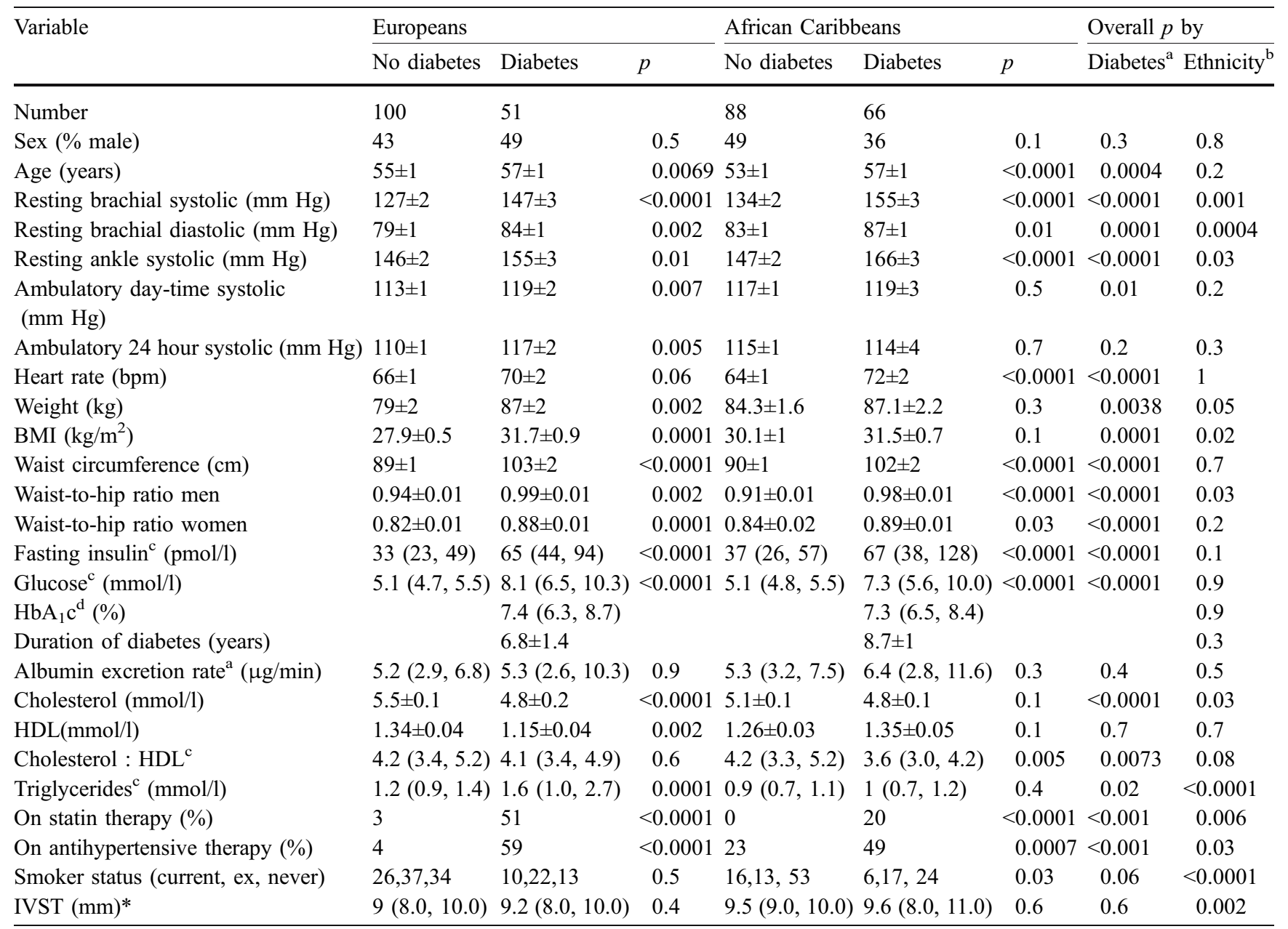

Values are means \pm SE, except skewed data where geometric mean, 25th and 75th percentile, are shown.

$p$ values are for significance between non-diabetic and diabetic group within an ethnic group, except ${ }^{\mathrm{a}}$ and $^{\mathrm{b}}$

${ }^{a}$ For difference between those with and without diabetes in the whole population

${ }^{\mathrm{b}}$ For difference between Europeans and African Caribbeans in the whole population

${ }^{\mathrm{c}}$ Log-transformed variable

${ }^{\mathrm{d}}$ Inverse-transformed variable 
a

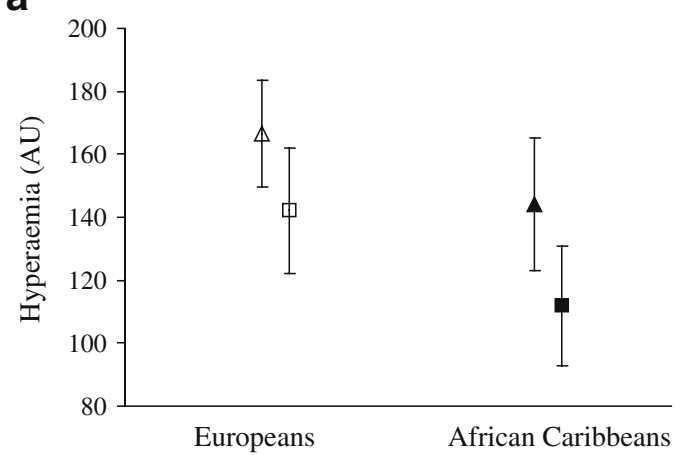

b

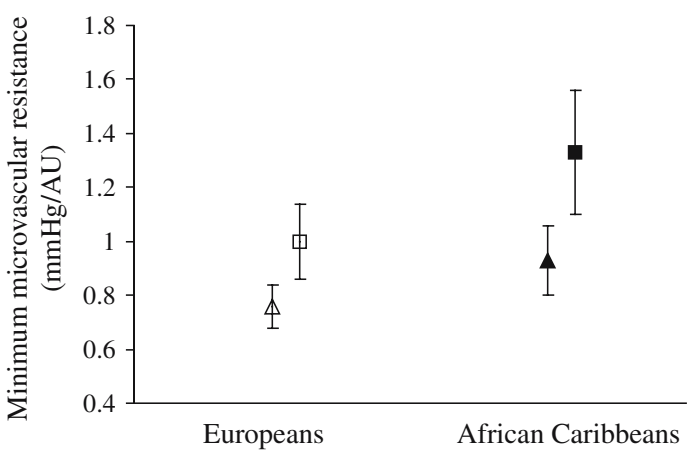

C

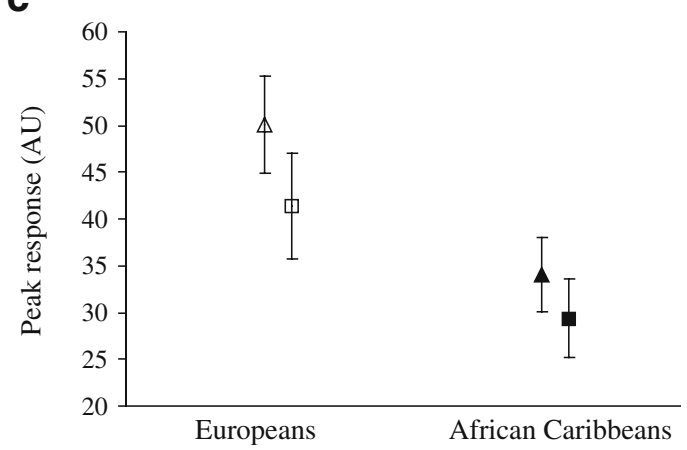

Fig. 1 The effects of diabetes on microvascular responses in each ethnic group. a Age-adjusted maximum hyperaemic response (arbitrary units) $\pm 95 \%$ CIs, stratified by ethnicity and diabetes status. Difference due to diabetes: $p=0.08$ for Europeans; $p=0.03$ for African Caribbeans. b Age-adjusted minimum microvascular resistance (mm Hg/arbitrary units) $\pm 95 \%$ CIs, stratified by ethnicity and diabetes status. Difference due to diabetes: $p=0.003$ for Europeans; $p=0.003$ for African Caribbeans. c Age-adjusted geometric mean of peak response to ischaemia (arbitrary units) $\pm 95 \%$ CIs, stratified by ethnicity and diabetes status. Difference due to diabetes: $p=0.01$ for Europeans; $p=0.1$ for African Caribbeans. Open triangles, Europeans with no diabetes; open squares, Europeans with diabetes; filled triangles, African Caribbeans with no diabetes; filled squares, African Caribbeans with diabetes

ference in blood pressure parameters, glycaemic control, or rates of end organ damage between subjects of Black African descent who were directly from West Africa and Black African subjects from the Caribbean, all of whom were first-generation migrants (data not shown). a

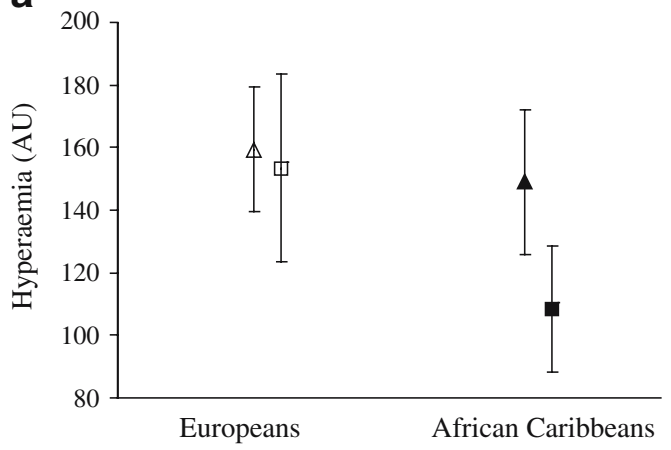

b

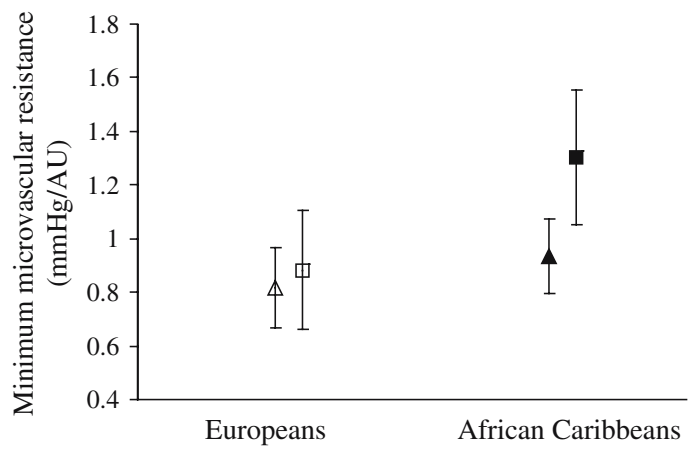

C

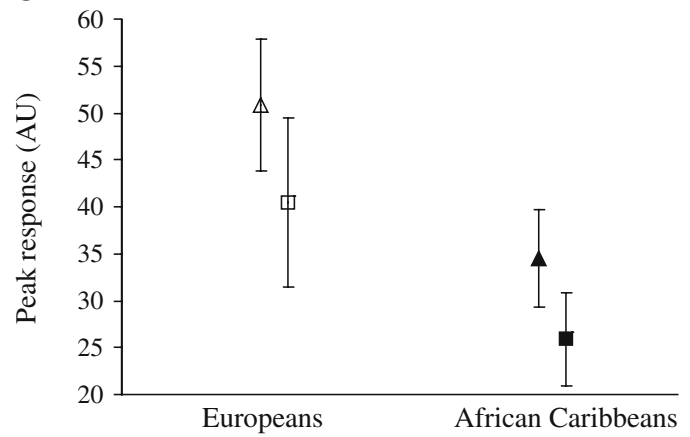

Fig. 2 The effect of diabetes on microvascular responses after full adjustment for potential mechanistic and confounding factors by ethnic specific multivariate analysis. a Fully adjusted maximum hyperaemia (arbitrary units) $\pm 95 \%$ CIs, stratified by ethnicity and diabetes status. Difference due to diabetes: $p=0.8$ for Europeans (adjusted for age, sex, resting diastolic BP, height, fasting glucose, HDL-cholesterol and statin therapy); $p=0.03$ for African Caribbeans (adjusted for age, sex, resting diastolic BP, waist-to-hip ratio, insulin, cholesterol : HDL-cholesterol ratio and statin therapy). b Fully adjusted minimum microvascular resistance (mm Hg/arbitrary units) $\pm 95 \%$ CIs, stratified by ethnicity and diabetes status. Difference due to diabetes: $p=0.2$ for Europeans (adjusted for age, sex, nocturnal ambulatory BP, BMI, fasting glucose, HDL-cholesterol and statin therapy); $p=0.02$ for African Caribbeans (adjusted for age, sex, resting diastolic BP, waist-to-hip ratio, insulin, cholesterol:HDL-cholesterol ratio and statin therapy). c Fully adjusted peak response to ischeamia (arbitrary units) $\pm 95 \%$ CIs, stratified by ethnicity and diabetes status. Difference due to diabetes: $p=0.2$ for Europeans (adjusted for age, sex, $24 \mathrm{~h}$ ambulatory BP, height, fasting glucose, total cholesterol and statin therapy); $p=0.05$ for African Caribbeans (adjusted for age, sex, $24 \mathrm{~h}$ ambulatory BP, waist-to-hip ratio, fasting glucose, triglycerides and statin therapy). Open triangles, Europeans with no diabetes; open squares, Europeans with diabetes; filled triangles, African Caribbeans with no diabetes; filled squares, African Caribbeans with diabetes 


\section{Microvascular measures}

The maximum hyperaemic response did not differ by diabetes status in Europeans $(p=0.08)$ but was markedly reduced in African Caribbeans with diabetes compared to those without ( $p=0.03$; Fig. 1a). The minimum microvascular resistance, which accounts for blood pressure differences, was higher in diabetic subjects in both ethnic groups, and higher in African Caribbeans than in Europeans, both in the diabetic $(p=0.003)$ and in the non-diabetic groups $(p=0.04)$ (Fig. 1b). The peak response to ischaemia was attenuated in the presence of diabetes in Europeans ( $p=0.03$ ) but not in African Caribbeans ( $p=0.5$ ) (Fig. 1c).

Maximum hyperaemia was strongly associated with glucose in Europeans only ( $\beta$ regression coefficient between log fasting glucose and log maximum hyperaemic response $-0.388 \pm 0.135, p=0.005$ ), and with $\mathrm{HbA}_{1} \mathrm{c}$ in African Caribbeans only ( $\beta$ between $\mathrm{HbA}_{1} \mathrm{c}^{-1}$ and $\log$ maximum hyperaemic response $7.40 \pm 2.9, p=0.01$ ), such that attenuated flow was associated with poorer diabetic control. Minimum microvascular resistance, in contrast, was associated with glucose in both ethnic groups $(\beta$ in Europeans $0.60 \pm 0.14, p<0.001$; in African Caribbeans $0.46 \pm 0.17, p=0.007)$, but again with $\mathrm{HbA}_{1} \mathrm{c}$ in African Caribbeans only ( $\beta$ in Europeans $-0.34 \pm 2.8, p=0.9$; in African Caribbeans $-8.23 \pm 2.9, p=0.007)$. Minimum microvascular resistance was associated with measures of obesity in Europeans only ( $p=0.01,0.006$ and 0.006 for weight, BMI and waist circumference, respectively), but not in African Caribbeans $(p=0.2,0.6,0.2)$. Peak response showed no clear association with any traditional measure of cardiovascular risk.

Explaining the effect of diabetes on microvascular response

As previously described, multivariate modelling was used to explore the mechanisms of the effect of diabetes on microvascular parameters. The lower maximal hyperaemia in Europeans with diabetes compared to those without was abolished when adjusted for brachial diastolic blood pres- sure, BMI, fasting glucose, HDL-cholesterol and statin therapy (159 arbitrary units [AU] [95\% CI 140-181] in those without diabetes vs 153 AU. [95\% CI 125-189] in those with diabetes, $p=0.8$ ) (Fig. $2 \mathrm{a}$ ). In contrast to the Europeans, the difference in maximum hyperaemia between African Caribbeans without and with diabetes persisted after multivariate adjustment (149 AU [95\% CI $127-$ 175] vs 108 AU [95\% CI 90-131], $p=0.03$ ). Similarly, the greater minimum microvascular resistance and lower peak response in Europeans with diabetes compared to those without was accounted for by cardiovascular risk factors ( $p=0.7$ and 0.2 , respectively), whereas in African Caribbeans with and without diabetes these differences could not be explained ( $p=0.02$ for minimum microvascular resistance; $p=0.05$ for peak response) (Fig. 2b and c, respectively). However, this difference in response did not reach statistical significance ( $p=0.09$ for interaction). When these analyses were repeated on patients taking no vasoactive medications (defined as anti-anginals, anti-hypertensives, sulphonylureas and insulin), the quantitative change in the differences was small, although the reduction in numbers attenuated the significance of the difference $(p=0.7$ and $p=0.06$ for maximum hyperaemia in Europeans and African Caribbeans respectively; $p=0.03$ for minimum microvascular resistance in both groups).

\section{Microvascular associations with IVST}

Maximum hyperaemia was inversely associated with IVST. As this was independent of ethnicity or sex, ethnic groups were combined for analysis. There was an interaction between diabetes and maximum hyperaemia on IVST $(p=0.03)$, such that in persons with diabetes, the association between IVST and maximum hyperaemia was negative, whereas in those without diabetes there was no such association (Table 2). The association with diabetes was independent of blood pressure, obesity, fasting insulin and lipid profiles. In a similar manner, minimum microvascular resistance in those with diabetes was positively associated with IVST independently of classical cardiovascular risk factors, such that the higher the resistance, the greater the
Table 2 Association between IVST and microvascular parameters after adjustment for age, sex and a diabetes-specific multivariate model of cardiovascular risk factors in the entire population

Table shows $\beta$ regression coefficients \pm SE

${ }^{a} p$ for contribution of the microvascular parameter to the model

${ }^{\mathrm{b}} R^{2}=$ variance explained by the whole model

\begin{tabular}{|c|c|c|c|c|c|c|}
\hline \multirow[t]{2}{*}{ IVST association with } & \multicolumn{3}{|l|}{ No diabetes } & \multicolumn{3}{|l|}{ With diabetes } \\
\hline & $\beta \pm \mathrm{SE}$ & $p^{\mathrm{a}}$ & $R^{2}(\%)^{\mathrm{b}}$ & $\beta \pm \mathrm{SE}$ & $p^{\mathrm{a}}$ & $R^{2}(\%)^{\mathrm{b}}$ \\
\hline Maximum hyperaemia & $-0.023 \pm 0.016$ & 0.2 & 1.2 & $-0.067 \pm 0.030$ & 0.03 & 4.7 \\
\hline Age and sex adjusted & $-0.013 \pm 0.016$ & 0.4 & 15.2 & $-0.074 \pm 0.029$ & 0.01 & 17.4 \\
\hline Multivariate model $^{\mathrm{a}}$ & $-0.007 \pm 0.012$ & 0.6 & 51.3 & $-0.062 \pm 0.025$ & 0.01 & 49.5 \\
\hline Minimum microvascular resistance & $0.047 \pm 0.016$ & 0.006 & 4.4 & $0.094 \pm 0.028$ & 0.001 & 10.1 \\
\hline Age and sex adjusted & $0.029 \pm 0.015$ & 0.06 & 16.7 & $0.086 \pm 0.027$ & 0.002 & 20.3 \\
\hline Multivariate model $^{\mathrm{a}}$ & $0.007 \pm 0.012$ & 0.6 & 51.3 & $0.058 \pm 0.024$ & 0.02 & 49.1 \\
\hline \multicolumn{7}{|l|}{ Diabetes-specific model } \\
\hline No diabetes & \multicolumn{6}{|c|}{$\begin{array}{l}\text { Age, sex, brachial systolic BP, HDL, fasting insulin, weight and } \\
\text { statin therapy }\end{array}$} \\
\hline With diabetes & \multicolumn{6}{|c|}{$\begin{array}{l}\text { Age, sex, ankle systolic BP, cholesterol: HDL ratio, fasting insulin, } \\
\text { waist circumference and statin therapy }\end{array}$} \\
\hline
\end{tabular}


IVST. Once again, this association was not observed in those without diabetes. There was no association between peak response and IVST (data not shown).

If a classical risk factor model was adopted, using resting systolic blood pressure, total cholesterol, fasting glucose and BMI, the association between microvascular responses and IVST, and the interaction between those with and without diabetes would persist, although the variance explained by the model falls ( $\beta$ for association between maximum hyperaemia and IVS after adjustment in those with diabetes $=-0.068 \pm 0.027, p=0.01 ; p=0.05$ for interaction between diabetes and maximum hyperaemia).

\section{Discussion}

We have shown, for the first time, that microvascular perfusion is deranged not only in Europeans but also in African Caribbeans with diabetes, compared to those without. In addition we have demonstrated that determinants of microvascular dysfunction in diabetes differ according to ethnicity. In our white European population, the effect of diabetes on microvascular function could be wholly accounted for by measures of insulin resistance, blood pressure and other cardiovascular risk factors. In African Caribbeans, however, conventional cardiovascular risk factors did not explain the effect of diabetes on the microvasculature. This has implications both for our understanding of the mechanisms of progression of microvascular disease, and for the therapeutic options.

The presence of retinopathy and cerebral white matter lesions, as markers of microvascular damage, are highly predictive of future stroke [27], and the presence of an altered albumin excretion rate is well known to be associated with future cardiovascular events down to levels that are currently thought of as normal $[28,29]$. However, these measures are either proxies for, or results of, microvascular damage. We chose to compare our measures of microvascular function with IVST as a measure of vascular target organ damage. Left ventricular mass index is not a valid proxy for left ventricular hypertrophy in these interethnic comparisons because of the alterations in left ventricular geometry seen in African Caribbeans compared to Europeans, such that African Caribbeans are more prone to concentric left ventricular hypertrophy, with a reduction in ventricular diameter, compared to the eccentric left ventricular hypertrophy seen in Europeans with an increase in ventricular diameter $[20,21]$. An association between microvascular measures and microvascular outcomes such as proteinurea or creatinine would be anticipated. However, the role of the microvasculature in vascular target organ damage such as left ventricular hypertrophy remains more controversial.

In Europeans, it is well known that diabetes precipitates microvascular damage and that this is strongly associated with conventional cardiovascular risk factors. In the UKPDS, and more latterly the Collaborative Atorvastatin Diabetes Study (CARDS), aggressive management of these risk factors, with tight glycaemic control [10], use of an- tihypertensives [9] and lipid management with statins [30] resulted in a reduced incidence of micro- and macrovascular complications [10, 31]. However, the population studied in the UKPDS was predominantly of white European origin, with only $8 \%$ of patients having a Black African ancestry [32], and CARDS had a 96\% Caucasian population [33]. Therefore the results of these studies may not be representative of the benefits of treating these cardiovascular risks in a multi-ethnic society. Type 2 diabetes is also associated with microvascular dysfunction in African Caribbeans. However, the role of conventional risk factors in disease development is not clear and cannot be assumed to be identical to those in Europeans. Populationbased studies demonstrate that, whereas ischaemic heart disease occurs less frequently [16], microvascular complications occur earlier and are more common in African Caribbeans [5] than in their European counterparts. Early reports suggested that ethnic differences in vascular target organ damage were due to earlier onset of diabetes in African Caribbeans, together with an increased incidence of hypertension, limited access to health care resulting in poorer glycaemic control, obesity, lower socioeconomic status, and lower education levels [6, 34]. However, in the Multiple Risk Factor Intervention Trial, these factors accounted for some, but not all of the differences in incidence of end-stage renal failure between the two ethnic groups, leaving some differences in microvascular outcomes unexplained [6]. This in turn suggests the presence either of alternative, ethnicity-dependent, mechanistic pathways for target organ damage, or of a different genetic predisposition to the effects of risk factors.

One clear ethnic difference is the prevalence of saltsensitive hypertension among African Caribbeans. Independent of its effect on blood pressure, salt sensitivity is known to have a direct effect on the microvasculature of Dahl salt-sensitive hypertensive rats, precipitating intense vasoconstriction and resulting in increased microvascular resistance in the proximal arterioles [35]. It is therefore reasonable to suggest that in salt-sensitive states, such as that pertaining to African Caribbeans, vasodilation within the microcirculatory bed should be impaired. In the presence of hypertension and vasoconstriction, endogenous vasodilators such as members of the natriuretic family would be expected to be upregulated. Contrary to expectations, however, Blacks with hypertension induced by salt sensitivity have a reduction in one of the most potent of these vasodilators, A-type natriuretic peptide [36], compared to Whites with and without salt sensitivity and salt-resistant Blacks. This may represent a failure of microvascular protective measures against the effect of hypertension, resulting in the observed increase in minimal microvascular resistance.

Of course, the role of genetic variation must also be considered. The inheritance of type 2 diabetes is likely to be polygenic [37]. Polymorphisms in the Calpain 10 gene on a region of chromosome $2 \mathrm{q} 37$ are associated with risk of developing type 2 diabetes [38]. The polymorphism that confers susceptibility to type 2 diabetes is associated with altered microvascular function [39]. Although the prevalence of this gene has not been studied in African Caribbeans to 
date, it is reasonable to expect that this or other similar genes may play a dual role in the high incidence of diabetes and attenuated microvascular function.

Our findings raise the possibility that in African Caribbeans microvascular dysfunction associated with type 2 diabetes may not be dependent on conventional cardiovascular risk factors.

If this is the case, it may be inappropiate to extrapolate results from large interventional clinical trials such as UKPDS and CARDS to populations outside the original ethnic group. The notion that similar blood pressure and glycaemic control may not have the same beneficial effects in these patients is in keeping with a recent report showing that, after adjustment for age, sex, geographic variations, cardiovascular disease and hypertension, African American veterans had a 30\% higher risk of diabetic nephropathy than white Americans, despite similar therapy and the same targets being achieved in both groups [40]. The investigation of relative beneficial impacts of existing therapies on microvascular function and identification of alternative novel cardiovascular risk factors present in African Caribbeans may have profound effects on future treatment strategies for these patients.

The possible limitations of this study include the fact that it focuses on the impact of diabetes on the skin microvasculature within two ethnic groups. To date, however, there is no direct evidence that these measures predict microvascular or macrovascular events in either ethnic group. We have demonstrated an ethnicity-independent association between skin microvascular function and IVST only in subjects with diabetes. Our principle hypothesis was that the effect of diabetes on skin microvasculature does not have the same determinants in African Caribbeans as in Europeans. Given this, it is also possible that the skin microvasculature does not have the same associations with target organ damage in the two ethnic groups.

\section{Conclusion}

We demonstrated attenuation of microvascular structure and function in diabetic patients from European and African Caribbean groups. In subjects of European descent, the attenuation due to diabetes was wholly accounted for by conventional cardiovascular risk factors, largely blood pressure and insulin. In African Caribbeans, however, conventional risk factors did not account for the impact of diabetes on the microcirculation. In diabetic subjects of both groups, this attenuation of microvascular function was strongly associated with left ventricular hypertrophy, independently of ethnicity and cardiovascular risk factors. The role of alternative risk factors in the pathogenesis of microvascular damage needs to be further investigated, paricularly in African Caribbeans, with a view to developing novel treatment strategies.
Acknowledgements We would like to thank the British Heart Foundation for funding this work and the general practitioners of the Law Medical Practice and the Brentfield Medical Centre, London, $\mathrm{UK}$, for their assistance in patient recruitment.

\section{References}

1. Gu K, Cowie CC, Harris MI (1999) Diabetes and decline in heart disease mortality in US adults. JAMA 281:1291-1297

2. Chaturvedi N, McKeigue PM, Marmot MG (1993) Resting and ambulatory blood pressure differences in Afro-Caribbeans and Europeans. Hypertension 22:90-96

3. Chaturvedi N, Jarrett J, Morrish N, Keen H, Fuller JH (1996) Differences in mortality and morbidity in African Caribbean and European people with non-insulin dependent diabetes mellitus: results of 20 year follow up of a London cohort of a multinational study. Br Med J 313:848-852

4. Chaturvedi N, McKeigue PM, Marmot MG, Nihoyannopoulos P (2001) A comparison of left ventricular abnormalities associated with glucose intolerance in African Caribbeans and Europeans in the UK. Heart 85:643-648

5. Cowie CC, Port FK, Wolfe RA, Savage PJ, Moll PP, Hawthorne VM (1989) Disparities in incidence of diabetic end-stage renal disease according to race and type of diabetes. N Engl J Med 321:1074-1079

6. Brancati FL, Whittle JC, Whelton PK, Seidler AJ, Klag MJ, (1992) The excess incidence of diabetic end-stage renal disease among blacks. A population-based study of potential explanatory factors. JAMA 268:3079-3084

7. Wong TY, Klein R, Couper DJ et al (2001) Retinal microvascular abnormalities and incident stroke: the Atherosclerosis Risk in Communities Study. Lancet 358:1134-1140

8. Ijzerman RG, de Jongh RT, Beijk MA et al (2003) Individuals at increased coronary heart disease risk are characterized by an impaired microvascular function in skin. Eur J Clin Invest 33:536-542

9. UKPDS 38 (1998) Tight blood pressure control and risk of macrovascular and microvascular complications in type 2 diabetes: UKPDS 38. UK Prospective Diabetes Study Group. Br Med J 317:703-713

10. UKPDS Group (1998) Effect of intensive blood-glucose control with metformin on complications in overweight patients with type 2 diabetes: UKPDS 34. UK Prospective Diabetes Study Group. Lancet 352:854-865

11. Chaturvedi N, Bulpitt CJ, Leggetter S et al (2004) Ethnic differences in vascular stiffness and relations to hypertensive target organ damage. J Hypertens 22:1731-1737

12. Rajkumar C, Mensah R, Meeran K, Armstrong S, Bulpitt CJ, (1999) Peripheral arterial compliance is lower in AfroCaribbeans compared to white Caucasians with type 2 diabetes after adjustment for blood pressure. J Hum Hypertens 13:841843

13. Strain WD, Chaturvedi N, Leggetter $S$ et al (2005) Ethnic differences in skin microvascular function and their relation to cardiac target-organ damage. J Hypertens 23:133-140

14. Bone M (1984) Registration with general medical practitioners in inner London: a survey carried out on behalf of the Department of Health and Social Security. HMSO, London

15. Akinkugbe OO (1985) World epidemiology of hypertension in Blacks. In: Hall WD, Saunders E, Shulman NB (eds) Hypertension in Blacks. Year Book Medical Publishers, Chicago, pp 3-16

16. Chaturvedi N, Fuller JH (1996) Ethnic differences in mortality from cardiovascular disease in the UK: do they persist in people with diabetes? J Epidemiol Community Health 50:137-139

17. Wild S, McKeigue P (1997) Cross sectional analysis of mortality by country of birth in England and Wales, 1970-92. Br Med J 314:705-710 
18. Chaturvedi N, McKeigue PM (1994) Methods for epidemiological surveys of ethnic minority groups. J Epidemiol Community Health 48:107-111

19. Devereux RB, Liebson PR, Horan MJ (1987) Recommendations concerning use of echocardiography in hypertension and general population research. Hypertension 9:97-104

20. Mayet J, Shahi M, Foale RA, Poulter NR, Sever PS, Thom SAM (1994) Racial differences in cardiac structure and function in essential hypertension. Br Med J 308:1011-1014

21. Chaturvedi N, Athanassopoulos G, McKeigue PM, Marmot MG, Nihoyannopoulos P (1994) Echocardiographic measures of left ventricular structure and their relation with rest and ambulatory blood pressure in blacks and whites in the United Kingdom. J Am Coll Cardiol 24:1499-1505

22. Kizer JR, Arnett DK, Bella JN et al (2004) Differences in left ventricular structure between black and white hypertensive adults: the Hypertension Genetic Epidemiology Network study. Hypertension 43:1182-1188

23. Vuilleumier P, Decosterd D, Maillard M, Burnier M, Hayoz D (2002) Postischemic forearm skin reactive hyperemia is related to cardiovascular risk factors in a healthy female population. J Hypertens 20:1753-1757

24. Williams SA, Tooke JE (1992) Noninvasive estimation of increased structurally-based resistance to blood flow in the skin of subjects with essential hypertension. Int J Microcirc Clin Exp 11:109-116

25. Cupples LA, Heeren T, Schatzkin A, Colton T (1984) Multiple testing of hypotheses in comparing two groups. Ann Intern Med 100:122-129

26. Rothman KJ (1990) No adjustments are needed for multiple comparisons. Epidemiology 1:43-46

27. Wong TY, Klein R, Sharrett AR et al (2002) Cerebral white matter lesions, retinopathy, and incident clinical stroke. JAMA 288:67-74

28. Kuusisto J, Mykkanen L, Pyorala K, Laakso M (1995) Hyperinsulinaemic microalbuminuria. A new risk indicator for coronary heart disease. Circulation 91:831-837
29. Hillege HL, Fidler V, Diercks GF et al (2002) Urinary albumin excretion predicts cardiovascular and noncardiovascular mortality in general population. Circulation 106:1777-1782

30. Colhoun H, Betteridge DJ, Durrington P et al (2004) The Collaborative Atorvastatin Diabetes Study (CARDS). Effectiveness of lipid lowering for the primary prevention of major cardiovascular events in diabetes. Presented at American Diabetic Association 64th Scientific Sessions. Orange County Convention Centre, Florida: Diabetes

31. BARI Group (2000) Seven-year outcome in the Bypass Angioplasty Revascularization Investigation (BARI) by treatment and diabetic status. J Am Coll Cardiol 35:1122-1129

32. UKPDS (1994) UK Prospective Diabetes Study: XII. differences between Asian, Afro-Caribbean and white Caucasian type 2 diabetic patients at diagnosis of diabetes. UK Prospective Diabetes Study Group. Diabet Med 11:670-677

33. Colhoun HM, Thomason MJ, Mackness MI et al (2002) Design of the Collaborative AtoRvastatin Diabetes Study (CARDS) in patients with type 2 diabetes. Diabet Med 19:201-211

34. Harris MI (1990) Non-insulin-dependent diabetes mellitus in Black and White Americans. Diabetes Metab Rev 6:71-90

35. Boegehold MA (2002) Microvascular structure and function in salt-sensitive hypertension. Microcirculation 9:225-241

36. Campese VM, Tawadrous M, Bigazzi R et al (1996) Salt intake and plasma atrial natriuretic peptide and nitric oxide in hypertension. Hypertension 28:335-340

37. Cook JT, Shields DC, Page RC et al (1994) Segregation analysis of NIDDM in Caucasian families. Diabetologia 37:1231-1240

38. Evans JC, Frayling TM, Cassell PG et al (2001) Studies of association between the gene for calpain-10 and type 2 diabetes mellitus in the United Kingdom. Am J Hum Genet 69:544-555

39. Shore AC, Evans JC, Frayling TM et al (2002) Association of calpain-10 gene with microvascular function. Diabetologia 45: 899-904

40. Young BA, Maynard C, Boyko EJ (2003) Racial differences in diabetic nephropathy, cardiovascular disease, and mortality in a national population of veterans. Diabetes Care 26:2392-2399 